\title{
Coagulation size of freezable water in poly(vinyl alcohol) hydrogels formed by different freeze/thaw cycle periods
}

\author{
Tatsuro Nakano and Takahiko Nakaoki
}

The coagulation size of freezable water in a poly(vinyl alcohol) (PVA) hydrogel was investigated as a function of the freeze/thaw cycle period using thermal analysis. The melting temperature of ice in the gel shifted to a lower temperature than that of normal ice. This temperature depression can be interpreted in terms of the coagulation size of freezable water in the gel. For longer freeze/thaw cycle periods, the coagulation size became larger. In addition, the weight content of freezable water estimated from the melting enthalpy of ice increased with an increasing length of the freeze/thaw period. These results suggested an expansion of the coagulation size of freezable water. As for the molecular structure of PVA, the nature of the hydrogen bonds was investigated for a PVA/deuterated water $\left(D_{2} O\right)$ gel by infrared spectroscopy. A component analysis of the OD-stretching mode of $\mathrm{D}_{2} \mathrm{O}$ showed a decrease in multiple hydrogen bonds and an increase in the free OD-stretching mode over longer freeze/thaw periods. This led to the transformation from non-freezable water to freezable water. Therefore, it was concluded that the coagulation size of freezable water expands and the aggregation of PVA increases as a result of lengthening the freeze/thaw cycle.

Polymer Journal (2011) 43, 875-880; doi:10.1038/pj.2011.92; published online 21 September 2011

Keywords: coagulation size of water; freeze/thaw cycle; freezable water; gel; poly(vinyl alcohol)

\section{INTRODUCTION}

Poly(vinyl alcohol) (PVA) is a representative water-soluble and semicrystalline polymer that is known to be non-toxic to humans; it is used as a drug delivery system and as an artificial articular cartilage. ${ }^{1-5}$ A PVA gel can be prepared by chemical or physical crosslinking. Chemical crosslinking is generally achieved through covalent bonding through a chemical reaction or through $\gamma$-ray radiation. In contrast, physical crosslinking is formed by physical aggregation through crystallization or hydrogen bonding. It is well known that PVA forms a physically crosslinked hydrogel. To make a stable physical gel, Peppas et al. ${ }^{6}$ proposed the freeze/thaw cycle method. There have been many investigations of the molecular structure of the PVA gels formed through freeze/thaw cycles. ${ }^{7-10}$ In the initial stage of gelation, a spinodal liquid-liquid phase separation takes place, leading to the formation of a porous network structure with the pores mainly occupied by free water. The polymer-rich region consists of amorphous PVA swollen with water and a crystal that has a fringed, micelle-like structure. Coagulated free water is surrounded by swollen, amorphous PVA, and the crystalline aggregates functions as a crosslinking point. The degree of crystallinity has been reported to be as low as a few percent by X-ray scattering, ${ }^{1} \mathrm{H}$ NMR and thermal analysis. ${ }^{10}$

Most of the investigations of the PVA hydrogel were focused on the molecular structure at a crosslinking point, and there are few reports on water bound in the PVA gel. Thermal analysis was applied to investigate the bound state of water in the hydrogels, including PVA $^{11-14}$ and polysaccharides. ${ }^{15}$ The freezable water frozen in a PVA gel was investigated by Higuchi et al. ${ }^{12}$ They compared the freezing and melting enthalpies during the cooling and heating processes. Recently, we reported the coagulation size of freezable water in the gel prepared by a $20 \mathrm{~h} / 4 \mathrm{~h}$ freeze/thaw cycle by thermal analysis. ${ }^{10}$ The melting temperature of freezable water frozen in the gel is lower than that of normal ice. The temperature depression is closely related to the coagulation size and depends on the surface energy. The coagulation radius was $15.1 \mathrm{~nm}$ for a $10 \mathrm{wt} \%$ gel and became as small as $2.2 \mathrm{~nm}$ for a $50 \mathrm{wt} \%$ gel. Willcox et al. ${ }^{16}$ reported the microstructure of the PVA hydrogel produced by this cycling method. Cryogenic transmission electron microscopy provided direct evidence of the pore structure of the PVA hydrogel. The photographs showed rounded pores that were filled with water and had a diameter of $30 \mathrm{~nm}$ for a $10 \mathrm{wt} \%$ gel. This dimension is in good agreement with our previously reported value that was estimated by thermal analysis.

In the study reported here, the freeze/thaw cycle period was varied, and the coagulation size of freezable water bound in the PVA gel was evaluated by observing the melting behavior of ice in the gel. In addition, the aggregation of PVA chains was investigated by infrared spectroscopy. 


\section{EXPERIMENTAL PROCEDURE}

\section{Sample preparation}

PVA with a saponification degree of $98.5 \mathrm{~mol} \%$ was provided by Kuraray (Tokyo, Japan). The stereoregularity was determined to be $\mathrm{mm}=0.23, \mathrm{mr}=0.48$ and $\mathrm{rr}=0.29$ by ${ }^{1} \mathrm{H}$ NMR measurements. ${ }^{17}$ A PVA gel was formed by a freeze/ thaw cycle between freezing at $-20^{\circ} \mathrm{C}$ and thawing at $25^{\circ} \mathrm{C}$ after the homogeneous solution was formed at $120^{\circ} \mathrm{C}$ in an autoclave. The freeze/thaw cycle periods adopted were as following: $15 / 15 \mathrm{~min}, 30 / 30 \mathrm{~min}, 60 / 60 \mathrm{~min}$ and 120/120 min. The freezing and thawing were repeated twice.

Porous silica gels were purchased from Sigma-Aldrich Japan (Tokyo, Japan) and Fuji Silysia Chemical (Tokyo, Japan). The pore radii were 4.5, 7.3 and $8.6 \mathrm{~nm}$ for the Sigma-Aldrich material and $22.4 \mathrm{~nm}$ for that supplied by Fuji Silysia.

\section{Measurements}

Differential scanning calorimetry was performed using a Rigaku 8230D instrument (Rigaku, Tokyo, Japan). A small piece of gel of approximately $10 \mathrm{mg}$ was cut out and placed into the aluminum cell. The measurements were taken from $-100^{\circ} \mathrm{C}$ to $30^{\circ} \mathrm{C}$. The heating rate was $5^{\circ} \mathrm{C} \mathrm{min}-1$.

Attenuated total reflection-Fourier transform infrared spectroscopy was performed on a Jasco FT-IR 660 Plus spectrometer (Jasco, Tokyo, Japan) with a DTGS detector using a KRS-5 crystal. The gel sample was put onto the KRS-5 crystal. The measurements were taken at room temperature. A total of 32 transients were collected for each spectrum with a resolution of $2 \mathrm{~cm}^{-1}$.

\section{RESULTS AND DISCUSSION}

Relationship between the coagulation radius of freezable water and the melting temperature depression

The melting temperature of ice has a close relationship with the coagulation size. The surface energy is an important factor in defining the melting temperature. Hence, a small coagulation size results in a lower melting temperature because of the small surface energy. Ishikiriyama et al. ${ }^{18,19}$ have reported the relationship between the coagulation radius and the melting temperature depression of ice. They adopted the differential scanning calorimetry peak maximum temperature as a means of determining the temperature depression. However, a large amount of ice, which provides a large melting enthalpy, causes the peak maximum temperature to move to a higher temperature. Therefore, we attempted to redefine the relationship between the coagulation size and the onset temperature of ice melting using porous silica gels, whose pore radius is given in the Experimental Procedure section.

Figure 1 shows the differential scanning calorimetry data for icefilling pores of various radii in silica gel. In addition to the melting peak due to normal ice at $0{ }^{\circ} \mathrm{C}$, a second melting peak was clearly observed at a lower temperature. This melting temperature shifted to lower values as the pore size of the silica gel decreased. The drop in the onset temperature of melting, defined by the extrapolated dashed lines in Figure 1, is plotted in Figure 2. This low temperature shift is related to the coagulation size of the ice. The relationship between the pore radius of the silica gel $(R)$ and the temperature depression from the normal ice value $(\Delta T)$ is expressed as follows:

$$
R=\frac{\alpha(T)}{\Delta T}+\beta
$$

where $\alpha(T) / \Delta T$ and $\beta$ correspond to the pore radius of freezable water and the layer of non-freezable water, respectively. Following the previous report, ${ }^{18,19}(R-\beta) \Delta T$, corresponding to $\alpha(T)$, was plotted against $\Delta T$ as shown in Figure 3. Finally, $\alpha(T)$ was fitted by the following equation:

$$
\alpha(T)=-0.083 \Delta T+59.7
$$

The first term of equation (1) corresponds to the pore radius of freezable, bound solvent. This equation was applied to determine the coagulation radius of the freezable solvent in the PVA gel.

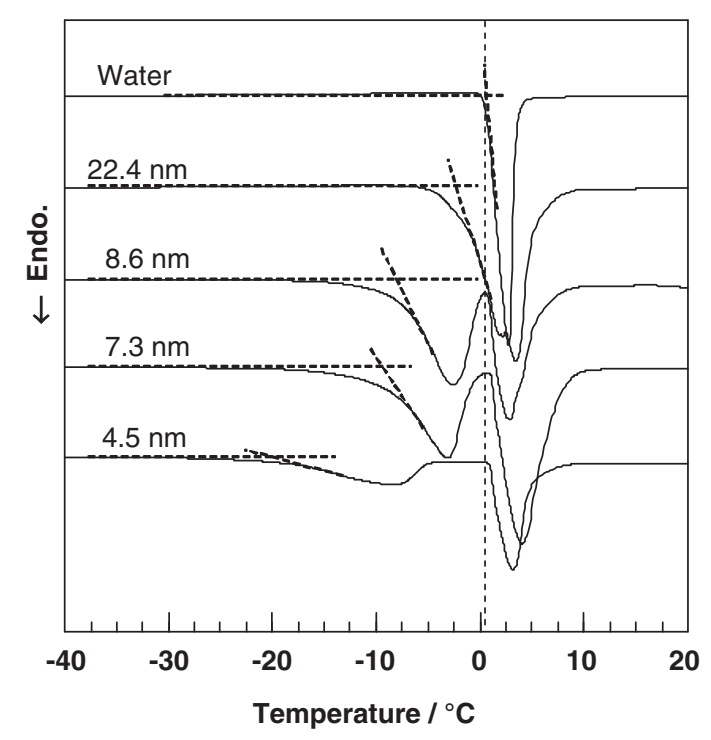

Figure 1 Melting behavior of ice trapped in porous silica gel observed by differential scanning calorimetry. The average pore radius is shown. In addition to the melting of normal ice at approximately $0{ }^{\circ} \mathrm{C}$, the endothermic peak due to the ice trapped in the pores of the silica gel was observed at lower temperatures.

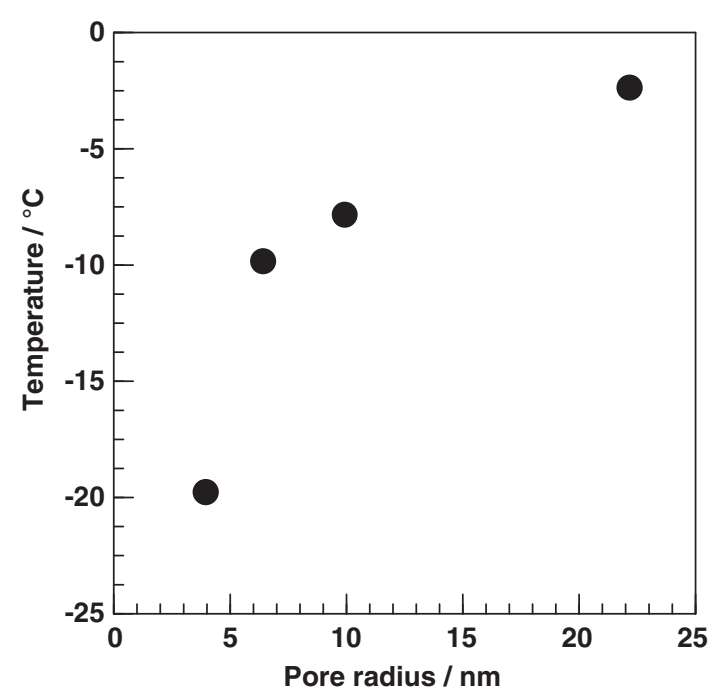

Figure 2 Melting temperature of ice in porous silica gel as a function of pore radius. The melting temperature depression decreased for silica gels with large pores.

Dependence of the coagulation size of freezable water in the PVA gel on the freeze/thaw period

The freezing and thawing period is an important factor in the formation of a stable gel, hence the dependence of the gelation process on the freezing and thawing period was investigated from the viewpoint of the coagulation size of freezable water in the gel. Figure 4 shows differential scanning calorimetry data for the melting of ice in the gel for various freeze/thaw cycle periods at four concentrations. The melting temperature for all gels was lower than that of normal ice. This indicates that the freezable water is bound in PVA chains and that the coagulation of freezable water varied in size depending on the freeze/thaw cycle period. The temperature depression from normal ice 


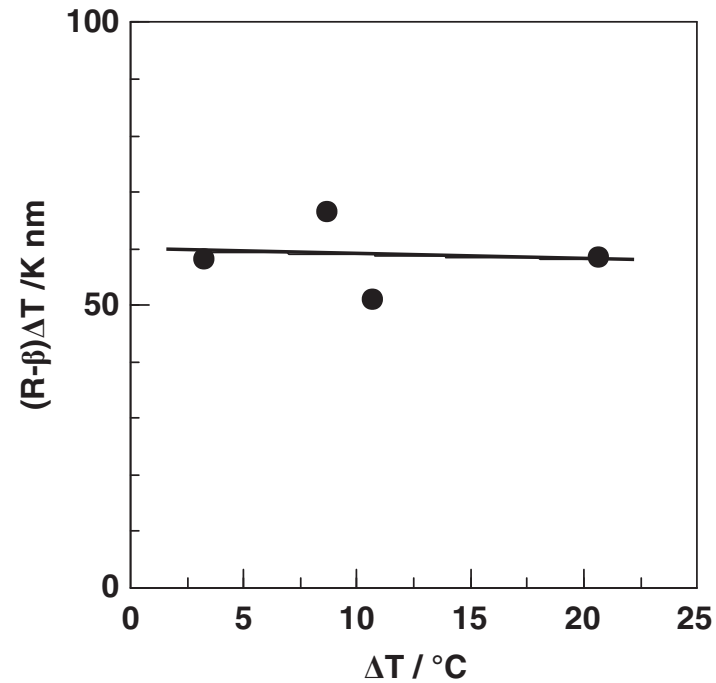

Figure 3 Plot of $(R-\beta) \Delta T$ corresponding to $\alpha(T)$ as a function of melting temperature depression.
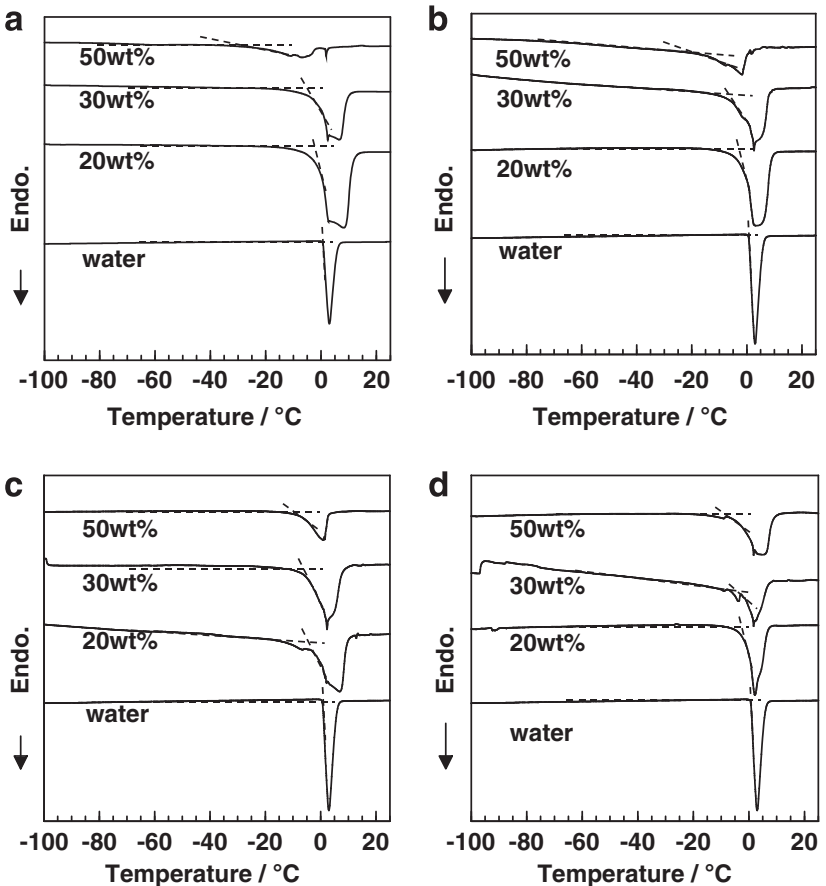

Figure 4 Melting behavior of ice in a poly (vinyl alcohol) PVA gel formed from different freeze/thaw cycle periods; (a) $15 / 15 \mathrm{~min}$, (b) $30 / 30 \mathrm{~min}$, (c) $60 / 60 \mathrm{~min}$ and (d) $120 / 120 \mathrm{~min}$.

is plotted against the freeze/thaw cycle period in Figure 5. A short freeze/thaw period resulted in a large temperature depression as compared with a longer freeze/thaw period. As described in the previous section, this temperature depression can be interpreted as being due to the coagulation size of ice in the gel. The coagulation radius of ice was estimated by equation (1) and is plotted in Figure 6. A longer freeze/thaw cycle period produced a larger coagulation radius of ice. For example, the coagulation radius for a $20 \mathrm{wt} \%$ gel with the freeze/thaw cycle of $15 / 15 \mathrm{~min}$ was $16 \mathrm{~nm}$, whereas it was $23 \mathrm{~nm}$ for a freeze/thaw cycle of $120 / 120 \mathrm{~min}$. To achieve this increase in coagulation radius, some non-freezable water must have changed to freezable

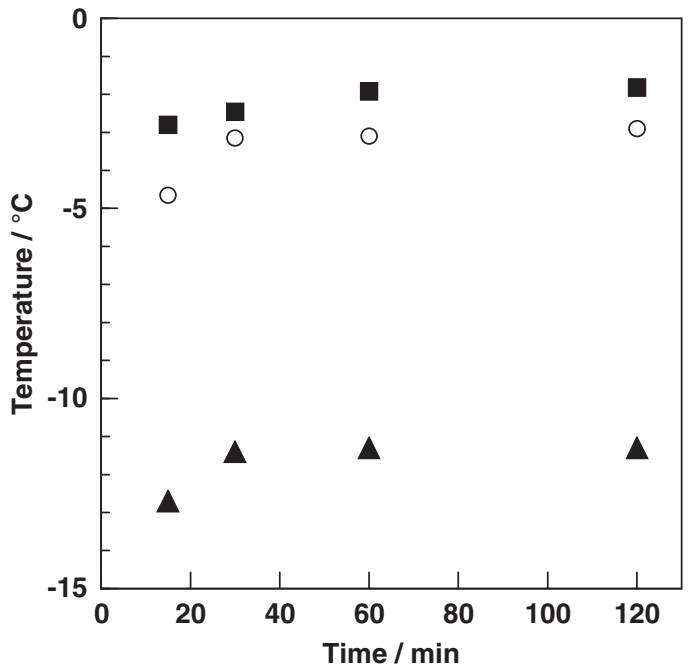

Figure 5 Melting temperature depression of ice bound in the poly(vinyl alcohol) PVA gel as a function of the freeze/thaw cycle period; $\mathbf{\square}: 20 \mathrm{wt} \%$, O: $30 \mathrm{wt} \%$ and $\mathbf{\Delta}: 50 \mathrm{wt} \%$.

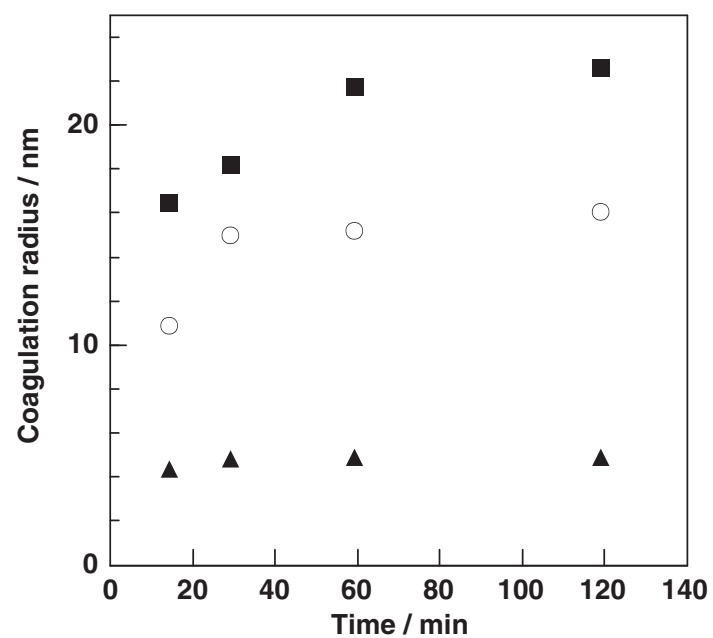

Figure 6 Coagulation radius of freezable water as a function of the freeze/ thaw cycle period; $\mathbf{\square}: 20 \mathrm{wt} \%, \mathrm{O}: 30 \mathrm{wt} \%$ and $\mathbf{\Lambda}: 50 \mathrm{wt} \%$.

water through transference from the swollen, amorphous PVA. Because of this, it is important to know the weight contents of freezable and non-freezable water; this will be evaluated in the next section.

\section{Weight content of freezable and non-freezable water}

There exist two states of freezable bound and non-freezable water in the PVA gel. The weight content of freezable water was estimated by the melting enthalpy observed in the gel. The rest of the water, which provides no melting enthalpy, can be assumed to be non-freezable water. Because the melting temperature shifted to lower temperatures, the melting enthalpy of supercooled water must be calibrated by the difference of heat capacities between solid ice and liquid water. The melting enthalpy, $\Delta H$, of supercooled water with a melting temperature $T$ is provided by the following equation:

$$
\Delta H(T)=\Delta H(273)-\int_{T}^{273} \Delta C_{p} \mathrm{~d} T
$$


where $\Delta H(273)$ is the melting enthalpy of normal water and $\Delta C_{\mathrm{p}}$ is the difference of heat capacity between solid ice and liquid water. ${ }^{20}$ This $\Delta H(T)$ is approximately expressed by the equation:

$$
\Delta H(T)=334.1+2.119 \cdot \Delta T-0.00783 \cdot \Delta T^{2}
$$

The weight content of freezable water can be estimated using the observed melting enthalpy, $\Delta \mathrm{H}_{\mathrm{F}}{ }^{\text {obs }}$

$$
w_{F}=\frac{\Delta H_{F}^{\text {obs }}}{\Delta H(T)}
$$

Then, the weight content of non-freezable water is expressed as follows:

$$
w_{N}=1-w_{F}
$$

Figure 7 shows the weight content of freezable water as a function of the freeze/thaw cycle period. The weight content of freezable water increased with increasing cycle period. This indicates that the water changed from non-freezable to freezable state when the cycle period was longer, which allows the coagulation size of the freezable water to expand. As for the concentration dependence at the same freeze/thaw cycle period, the weight content of freezable water was higher for a lower concentration gel. Because most of the water in a high-concentration gel is bound in the swollen, amorphous PVA, the content of freezable water would be correspondingly low.

According to the previous report, ${ }^{7}$ the coagulating freezable water is surrounded by swollen, amorphous PVA containing nonfreezable water. Therefore, the expansion of the coagulation size of freezable water would correlate with the shrinkage of the PVA domain, which can be realized by the crystallization and/or the aggregation of PVA chains through hydrogen bonding. Therefore, the presence of hydrogen bonding in the PVA chains was investigated by infrared spectroscopy.

\section{Infrared spectral analysis of the hydrogen bond between PVA and water}

The OH-stretching mode observed in the infrared spectrum is a suitable band for monitoring the presence of hydrogen bonds. To clarify the $\mathrm{OH}$-stretching mode for water, deuterated water $\left(\mathrm{D}_{2} \mathrm{O}\right)$ was used as a solvent. Figure 8 shows the OD-stretching mode of $\mathrm{D}_{2} \mathrm{O}$ for different freeze/thaw cycle periods. The peak intensity at approxi-

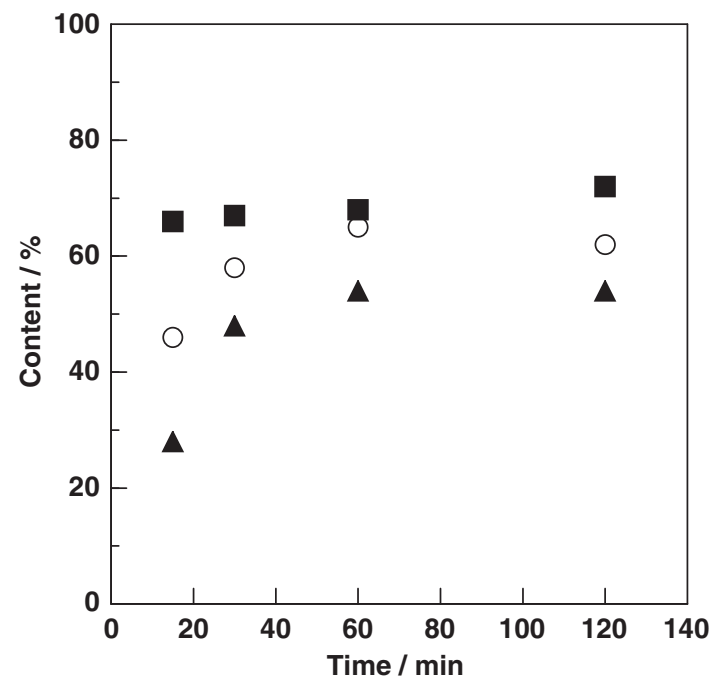

Figure 7 Weight content of freezable water as a function of the freeze/thaw cycle period; : 20 wt\%, O: 30 wt $\%$ and $\boldsymbol{\Lambda}: 50$ wt\%. mately $2400 \mathrm{~cm}^{-1}$ decreased. The OD mode can be separated into vibrations of three types of hydrogen-bonded species: the free OD mode at $2610 \mathrm{~cm}^{-1}$ and the OD modes with single and multiple hydrogen bonds at 2490 and $2390 \mathrm{~cm}^{-1}$, respectively. ${ }^{21-24}$ A typical curve fitting analysis is shown in Figure 9. The normalized intensity is plotted in Figure 10. Contrary to the OH mode of PVA, the free OD mode at $2610 \mathrm{~cm}^{-1}$ increased and the multiple OD modes decreased in intensity with increasing freeze/thaw cycle period length. This indicates that the hydrogen bonding in water is weaker for longer freeze/thaw cycle periods. This water can be assumed to be the freezable water. Therefore, the non-freezable water in the swollen, amorphous region of the PVA transformed to freezable water, which has comparatively weak hydrogen bonds.

These results are in good agreement with the results of the coagulation size and the weight content of freezable water found by thermal analysis. The process is shown schematically in Figure 11. In the initial stage of gelation, coagulated freezable water, the PVA domain of the swollen, amorphous material containing non-freezable water and crystallites are formed. In a gel formed by a long freeze/thaw cycle period, the coagulation size of freezable water became larger. As a result, the non-freezable water in the swollen, amorphous region of

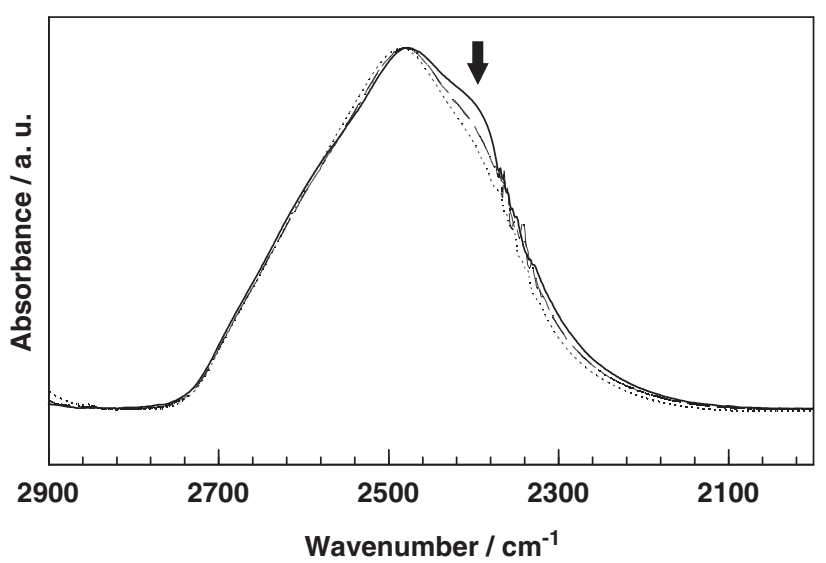

Figure 8 Infrared spectra of OD-stretching modes for deuterated water $\left(D_{2} \mathrm{O}\right)$ for different freeze/thaw cycle periods; $\longrightarrow$ : $15 / 15 \mathrm{~min},----$ : $30 /$ $30 \mathrm{~min}$, ...... : 60/60 min and .....: 120/120 min.

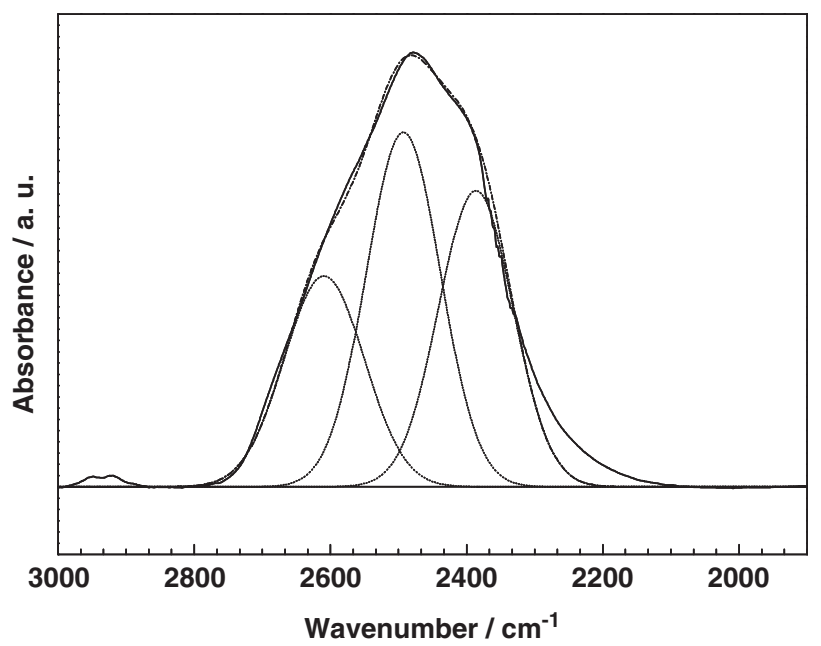

Figure 9 Curve fit analysis of and OD-stretching modes for deuterated water $\left(\mathrm{D}_{2} \mathrm{O}\right)$. 


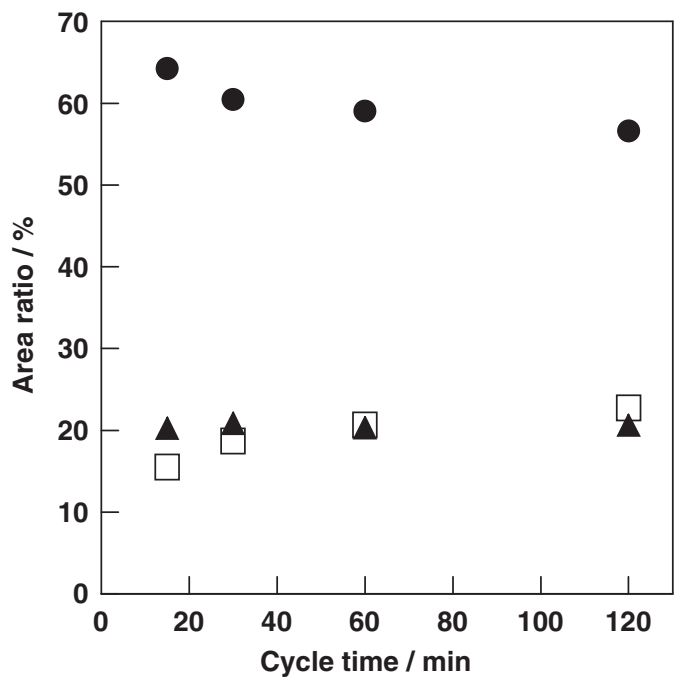

Figure 10 Integral intensities of the OD-stretching mode with various states of hydrogen bonding for deuterated water $\left(\mathrm{D}_{2} \mathrm{O}\right)$; $\boldsymbol{\Delta}$ : free mode, $\bullet$ : single hydrogen bond and $\square$ : multiple hydrogen bonds a

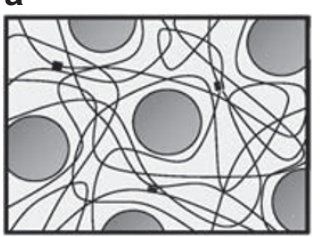

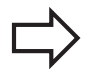

b

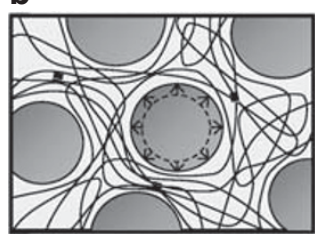

Figure 11 Schematic model of the gel morphology for short (a) and long (b) freeze/thaw cycle periods. At an early stage, a mixture of coagulated freezable water, swollen, amorphous PVA containing non-freezable water, and small PVA crystallites are formed. For a longer freeze/thaw cycle period, the aggregation of the PVA chain due to hydrogen bonding allows the coagulation size of freezable water to expand.

the PVA transformed to freezable water, allowing the domain of the freezable water to expand.

\section{CONCLUSION}

The coagulation size of freezable water in a PVA hydrogel was investigated as a function of the freeze/thaw cycle period by thermal analysis. An endothermic peak due to the melting of ice in the gel shifted toward lower temperatures than that of normal ice. This low temperature shift can be interpreted in terms of the coagulation size of ice. An equation relating the temperature depression and the coagulation size of ice was evaluated using porous silica gel with different pore sizes. This relationship was applied to determine the coagulation size of freezable water as a function of the freeze/thaw period. For longer freeze/thaw periods, the coagulation radius of freezable water became larger. The observed melting enthalpy corresponds to the content of freezable water. For longer freeze/thaw cycle periods, the content of freezable water increased, whereas that of non-freezable water decreased. We concluded from these thermal analyses that the coagulation size of freezable water was expanded for longer freeze/thaw cycle periods. Because the freezable water is surrounded by swollen, amorphous PVA, the PVA domain should shrink for longer freeze/ thaw cycle periods. The hydrogen bonding of the PVA chains was then investigated for the $\mathrm{PVA} / \mathrm{D}_{2} \mathrm{O}$ gel using infrared spectroscopy. The OD-stretching mode is very sensitive to hydrogen bonding and is separated into three components: the free stretching mode, and the stretching modes for single and multiple hydrogen bonds. The intensity of the free OD mode increased and that of the OD mode with multiple hydrogen bonds decreased for longer freeze/thaw cycle periods. This corresponds to the transformation of non-freezable water to freezable water. As a result, the coagulation size of freezable water expanded.

\section{ACKNOWLEDGEMENTS}

This work was partially supported by a grant from the High-Tech Research Center Program for private universities by the Japan Ministry of Education, Culture, Sports, Science and Technology. We also thank Dr Ohgi of Kuraray, who kindly supplied us with the PVA sample.

1 Tamura, K., Ike, O., Hitomi, S., Isobe, J., Shimizu, Y. \& Nambu, M. A new hydrogel and its medical application. Trans. Am. Soc. Artif. Intern. Organs 32, 605-608 (1986).

2 Oka, M., Nouguchi, T., Kumar, P., Ikeuchi, K., Yamamuro, T., Hyon, S. H. \& Ikada, Y. Development of an artificial articular cartilage. Clin. Mater. 6, 361-381 (1990).

3 Noguchi, T., Yamamuro, T., Oka, M., Kumar, P., Kotoura, Y., Hyon, S. H. \& Ikada, Y. Poly(vinyl alcohol) hydrogel as an artificial articular cartilage: evaluation of biocompatibility. J. Appl. Biomater. 2, 101-107 (1991).

4 Cha, W. I., Hyon, S. H. \& Ikada, Y. Mechanical and wear properties of poly(vinyl alcohol) hydrogels. Macromol. Symp. 109, 115-126 (1996).

5 Hassan, C. M. \& Peppas, N. A. Structure and applications of poly(vinyl alcohol) hydrogels produced by conventional crosslinking or by freezing/thawing methods. Adv. Polym. Sci. 153, 37-65 (2000).

6 Peppas, N. A. Turbidimetric studies of aqueous poly(vinyl alcohol) solutions. Makromol. Chem. 176, 3433-3440 (1975).

7 Ricciardi, R., Auriemma, F., De Rosa, C. \& Laupletre, F. X-ray diffraction analysis of poly(vinyl alcohol) hydrogels obtained by freezing and thawing techniques. Macromolecules 37, 1921-1927 (2004)

8 Ricciardi, R., Auriemma, F., Gaillet, C., De Rosa, C. \& Laupretre, F. Investigation of the crystallinity of freeze/thaw poly(vinyl alcohol) hydrogels by different techniques. Macromolecules 37, 9510-9516 (2004).

9 Ricciardi, R., Mangiapia, G., Lo Celso, F., Paduano, L., Triolo, R., Auriemma, F., De Rosa, C. \& Laupretre, F. Structural organization of poly(vinyl alcohol) hydrogels obtained by freezing and thawing techniques: a SANS Study. Chem. Mater. 17, 1183-1189 (2005)

10 Nakaoki, T. \& Yamashita, H. Bound states of water in poly(vinyl alcohol) hydrogel prepared by repeated freezing and melting method. J. Mol. Struct. 875, 282-287 (2008).

11 Hatakeyama, T., Yamaguchi, A. \& Hatakeyama, H. Studies on bound water in poly(vinyl alcohol) hydrogel by DSC and FT-NMR. Eur. Polym. J. 20, 61-64 (1984).

12 Higuchi, A. \& lijima, T.D.S.C. Investigation of the states of water in poly(vinyl alcohol) membranes. Polymer 26, 1207-1211 (1985).

13 Hatakeyama, T., Yamauchi, A. \& Hatakeyama, H. Effect of thermal hysteresis on structural change of water restrained in poly(vinyl alcohol) pseudo-gel. Eur. Polym. 23, 361-365 (1987).

$14 \mathrm{Li}$, W., Xue, F. \& Cheug, R. States of water in partially swollen poly(vinyl alcohol) hydrogels. Polymer 46, 12026-12031 (2005).

15 Hatakeyama, T., Quinn, F. X. \& Hatakeyama, H. Changes in freezing bound water in water-gellan systems with structure formation. Carbohydrate Polym. 30, 155-160 (1996).

16 Willcox, P. J., Howie, D. W., Shimidt-Rohr, K., Hoagland, D. A., Gido, S. P. Pudjijanto, S., Kleiner, L. W. \& Venkatraman, S. Microstructure of poly(vinyl alcohol) hydrogels produced by freeze/thaw cycling. J. Polym. Sci. Part B 37, 3438-3454 (1999).

17 Moritani, T., Kuruma, I. \& Shibatani, K. Tacticity of poly(vinyl alcohol) studied by nuclear magnetic resonance of hydroxyl protons. Macromolecules 5, 577-580 (1972).

18 Ishikiriyama, K., Todoki, M. \& Motomura, K. Pore size distribution(PSD) measurements of silica gels by means of differential scanning calorimetry. i. optimization for determination of PSD. J. Colloid and Interface Sci. 171, 92-102 (1995).

19 Ishikiriyama, K. \& Todoki, M. Pore size distribution measurements of silica gels by means of differential scanning calorimetry. ii. Thermoporosimetry. J. Colloid Interface Sci. 171, 103-111 (1995).

20 Angell, C. A., Shuppert, J. \& Tucker, C. Anomalous properties of supercooled water, heat capacity, expansivity, and proton magnetic resonance chemical shift from 0 to -38\%. J. Phys. Chem. 77, 3092-3099 (1973). 
21 Rull, F. Structural investigation of water and aqueous solutions by raman spectroscopy. Pure Appl. Chem. 74, 1859-1870 (2002).

22 Quack, M., Schmitt, U. \& Suhm, M. A. FTIR spectroscopy of hydrogen fluoride clusters in synchronously pulsed supersonic jets, isotopic isolation, substitution and 3-d condensation. Chem. Phys. Lett. 269, 29-38 (1997).
23 Quack, M., Stohner, J. \& Suhm, M. A. Analytical three-body interaction potentials and hydrogen bond dynamics of hydrogen fluoride aggregates (HF)n, $n \geqslant 3$. J. Mol. Struct. 599, 381-425 (2001).

24 Boychenko, I. V. \& Huber, H. Beyond the resonant dipole interaction model: resolution of a discrepancy between experimental and calculated structures of the carbon dioxide planar trimer. J. Chem. Phys. 124, 014305 (2006). 